\title{
A Vector Algebra Formulation of Mobile Robot Velocity Kinematics
}

\author{
Alonzo Kelly and Neal Seegmiller
}

\begin{abstract}
Typical formulations of the forward and inverse velocity kinematics of wheeled mobile robots assume flat terrain, consistent constraints, and no slip at the wheels. Such assumptions can sometimes permit the wheel constraints to be substituted into the differential equation to produce a compact, apparently unconstrained result. However, in the general case, the terrain is not flat, the wheel constraints cannot be eliminated in this way, and they are typically inconsistent if derived from sensed information. In reality, the motion of a WMR is restricted to a manifold which more-or-less satisfies the wheel slip constraints while both following the terrain and responding to the inputs. To address these more realistic cases, we have developed a formulation of WMR velocity kinematics as a differential-algebraic system - a constrained differential equation of first order. This paper presents the modeling part of the formulation. The Transport Theorem is used to derive a generic 3D model of the motion at the wheels which is implied by the motion of an arbitrarily articulated body. This wheel equation is the basis for forward and inverse velocity kinematics and for the expression of explicit constraints of wheel slip and terrain following. The result is a mathematically correct method for predicting motion over non-flat terrain for arbitrary wheeled vehicles on arbitrary terrain subject to arbitrary constraints. We validate our formulation by applying it to a Mars rover prototype with a passive suspension in a context where ground truth measurement is easy to obtain. Our approach can constitute a key component of more informed state estimation, motion control, and motion planning algorithms for wheeled mobile robots.
\end{abstract}

Alonzo Kelly and Neal Seegmiller

Robotics Institute, Carnegie Mellon University, e-mail: $\{$ alonzo, nseegmiller $\} @ \mathrm{cmu}$. edu 


\section{Introduction}

Wheeled mobile robots (WMRs) are perhaps the most common configuration of terrestrial mobile robot, and although decades of research are behind us, little has been revealed about how to model them effectively in anything other than flat floor environments. The motion model of the robot is nonetheless central to pose estimation, control, and motion planning.

Unlike for their predecessors, manipulators, modeling the articulations of the mechanisms involved is not the fundamental issue. WMRs need to know how they move over the terrain and such models are intrinsically differential equations. For WMRs, these equations are also constrained, the constraints are nonholonomic, the system is almost always overconstrained to some degree, and even if it was not, the constraints are typically violated in ways that are only partially predictable. In this light, it is perhaps less surprising that so little has been written on this problem. While its importance is clear, its solution is less clear.

Our own historical approaches to the problem [6] have avoided the issues by formulating inputs in state space, where constraints (and constraint consistency) are not an issue. Terrain following was treated after the fact by integrating the unconstrained dynamics and then forcing the constraints to be satisfied in a separate optimization process. While this was adequate, it was hardly principled.

While service robots may operate exclusively in flat floor environments, almost any useful field robot will have to operate competently on uneven, sloped, and slippery terrain for extended periods of time. The first step toward competent autonomy in these conditions is the incorporation of faster-than-real-time models that predict the consequences of candidate actions well. Fast and accurate WMR models are therefore a fundamental problem and we propose a general approach to designing such models in this paper.

\subsection{Prior Work}

Muir and Newman published one of the earliest general approaches to kinematic modeling of wheeled mobile robots [10]. Following Sheth-Uicker conventions they assign coordinate systems and derive a graph of homogenous transforms relating wheel and robot positions. By differentiating cascades of transforms, Jacobian matrices are computed for each wheel (relating wheel and robot velocities) which are combined to form the "composite robot equation." They provide a "sensed forward" solution (in which the robot velocity is determined from sensed steer angles and wheel velocities) as well as an "actuated inverse" solution.

Several researchers extended this transformation approach to WMR kinematics modeling. Alexander and Maddocks proposed an alternative forward solution when rolling without slipping is impossible, derived from Coulomb's Law of friction [1]. Rajagopalan handled the case of inclined steering columns [11]. Campion et al. classified WMR configurations into five mobility types based on degrees of mobility 
and steerability, which they define [2]. Yet others proposed geometric approaches to WMR kinematics modeling [5][7].

However, these earlier approaches and analyses are limited to planar motion. More recently in 2005, Tarokh and McDermott published a general approach to modeling full 6-DOF kinematics for articulated rovers driving on uneven terrain [13]. Their approach resembles Muir and Newman in requiring the derivation of homogenous transform graphs and the differentiation of transforms to compute wheel Jacobians. Others have derived and simulated full-3D WMR kinematics on rough terrain with specific objectives, such as mechanisms that enable rolling without slipping [4][3], precise localization [8], and control of passively-steered rovers [12].

In contrast to prior transformation and geometric approaches, we derive the kinematics and constraint equations for WMR using vector algebra. This new approach is intuitive and, unlike [13], does not require differentiation. Our method for propagating velocities forward through a kinematic chain is a classical one that has also been used in robot manipulation [9].

\section{Kinematics of Wheeled Mobile Robots}

In the general case, a wheeled mobile robot may be articulated in various ways and it may roll over arbitrary terrain with any particular wheel lying either on or above the nominal terrain surface. Assuming terrain contact is assured by geometry or a suspension, there are two principal difficulties associated with wheeled mobile robot (WMR) kinematic modeling: nonlinearity and overconstraint. Nonlinearity occurs in steering control because trigonometric functions of the steer angles appear in the mapping between body and wheel velocities. Overconstraint can occur in estimation contexts where the set of $m>n$ measurements of velocities and/or steer angles lead to an inconsistent solution for the $n$ degrees of velocity freedom available in the vehicle state vector. This section develops solutions for both the control and estimation problems using a vector algebraic formulation.

We will first develop the basic kinematic relationships between a) the linear and angular velocity of a distinguished coordinate frame on the body of the mobile robot and b) the linear velocity of an arbitrarily positioned point corresponding to a wheel. In contrast to all prior work, we will formulate the transformation using vector algebra, leading to a very straightforward expression for even the general case.

\subsection{Transport Theorem}

The key element of the technique is a basic theorem of physics, commonly used in dynamics and inertial navigation theory. Known either as the Coriolis Equation or the Transport Theorem, it concerns the dependence of measurements in physics on the state of motion of the observer. The notation $\vec{u}_{a}^{b}$ will mean the vector quantity 
$u$ of frame $a$ with respect to frame $b$. Let the letter $f$ refer to a frame of reference associated with a fixed observer, whereas $m$ will refer to one associated with a moving observer. Due to their relative, instantaneous angular velocity $\vec{\omega}_{m}^{f}$, our observers would compute (or measure) different time derivatives of the same vector $\vec{v}$ that are related as follows:

$$
\left.\frac{d \vec{v}}{d t}\right|_{f}=\left.\frac{d \vec{v}}{d t}\right|_{m}+\vec{\omega}_{m}^{f} \times \vec{v}
$$

\subsection{Velocity Transformation}

Now, let these two frames have an instantaneous relative position of $\vec{r}_{m}^{f}$. Suppose that the moving observer measures the position $\vec{r}_{o}^{m}$ and velocity $\vec{v}_{o}^{m}$ of an object $o$, and we wish to know what the fixed observer would measure for the motion of the same object. The position vectors can be derived from vector addition thus:

$$
\vec{r}_{o}^{f}=\vec{r}_{o}^{m}+\vec{r}_{m}^{f}
$$

The time derivative of this position vector, computed in the fixed frame is:

$$
\left.\frac{d}{d t}\right|_{f}\left(\vec{r}_{o}^{f}\right)=\left.\frac{d}{d t}\right|_{f}\left(\vec{r}_{o}^{m}+\vec{r}_{m}^{f}\right)=\left.\frac{d}{d t}\right|_{f}\left(\vec{r}_{o}^{m}\right)+\left.\frac{d}{d t}\right|_{f}\left(\vec{r}_{m}^{f}\right)
$$

Now we can apply the Coriolis equation to the first term on the right to produce the general result for the transformation of apparent velocities of the object $o$ between two frames of reference undergoing arbitrary relative motion:

$$
\vec{v}_{o}^{f}=\vec{v}_{o}^{m}+\vec{v}_{m}^{f}+\vec{\omega}_{m}^{f} \times \vec{r}_{o}^{m}
$$

We have used the fact that, for any frames $a$ and $b,\left.\frac{d}{d t}\right|_{b}\left(\vec{r}_{a}^{b}\right)=\vec{v}_{a}^{b}$.

\subsection{Wheel Equation}

We allow the point around which a wheel may steer (frame $s$ ) to be offset from the contact point (frame $c$ ) between the wheel and the ground (Figure 1). In such a case, we can write the position vector for the wheel contact point as follows:

$$
\vec{r}_{c}^{w}=\vec{r}_{v}^{w}+\vec{r}_{s}^{v}+\vec{r}_{c}^{s}
$$

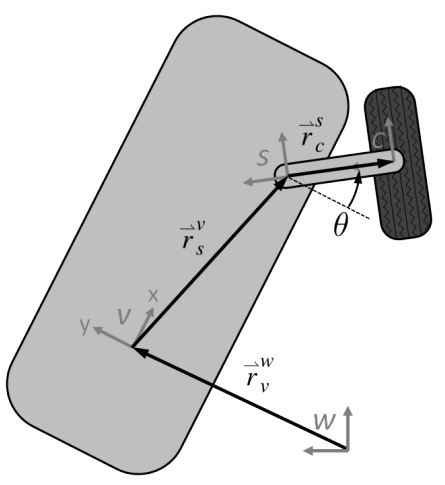

Fig. 1 Frames for WMR Kinematics. The four frames necessary for the relation of wheel rotation rates and to vehicle speed and angular velocity. 
Next, we associate any ground-fixed frame with the fixed observer and the bodyfixed frame with the moving observer and we can use the above velocity transformation to write a kinematic equation for each wheel. Differentiating the position vector in the world frame, substituting the Coriolis equation, and using $\vec{v}_{s}^{v}=0$ yields:

$$
\vec{v}_{c}^{w}=\vec{v}_{v}^{w}+\vec{\omega}_{v}^{w} \times \vec{r}_{s}^{v}+\vec{\omega}_{v}^{w} \times \vec{r}_{c}^{s}+\vec{\omega}_{c}^{v} \times \vec{r}_{c}^{s}
$$

This is important enough to give it a name: the wheel equation. In the case of no offset, the last two terms vanish and the steer velocity $\left(\vec{\omega}_{s}^{v}\right.$ or $\left.\vec{\omega}_{c}^{v}\right)$ no longer matters. The formula is valid in 3D and it also applies to cases with arbitrary articulations between the $v$ and $s$ frames because only the vector $\vec{r}_{s}^{v}$ is relevant. In other words, this is the general case.

\subsection{Inverse Velocity Kinematics - Body to Wheels}

Let the term inverse kinematics refer to the problem, relevant to control, of computing the wheel velocities from the body velocity. Given the above, the problem is solved by writing a wheel equation for each wheel. To do so, the physical vectors $\vec{u}$ must be expressed in a particular coordinate system. Let ${ }^{c} \underline{u}_{a}^{b}$ denote the vector quantity $u$ of frame $a$ with respect to frame $b$, expressed in the coordinates of frame $c$ (and let $\underline{u}_{a}^{b}$ imply ${ }^{b} \underline{u}_{a}^{b}$ ). Then, if $R_{s}^{v}$ is the rotation matrix that converts coordinates from the steer frame to the vehicle frame, it becomes possible to express the wheel equation for any wheel in the vehicle frame where many of the vectors are typically known:

$$
{ }^{v} \underline{v}_{c}^{w}={ }^{v} \underline{v}_{v}^{w}+{ }^{v} \underline{\omega}_{v}^{w} \times \underline{r}_{s}^{v}+{ }^{v} \underline{\omega}_{v}^{w} \times R_{s}^{v} \underline{r}_{c}^{s}+\underline{\omega}_{c}^{v} \times R_{s}^{v} \underline{r}_{c}^{s}
$$

\subsection{Wheel Steering and Drive - Control and Estimation}

In a control context, the wheel equation cannot be used directly as written to find wheel controls because the matrix $R_{s}^{v}$ depends on the steer angle, which is one of the unknowns. However, the steer angle can be found by expressing the wheel velocity in wheel coordinates and enforcing the constraint that the lateral (y) component of the terrain relative velocity in the wheel frame must vanish. For the geometry in Figure 1, the result is intuitive, the steer angle can be determined from the direction of the $s$ frame because its velocity is parallel to that of $c$, though not necessarily of the same magnitude. The velocity of frame $s$ is simply the first two terms of the wheel equation. Then, the steer angle for the wheel is:

$$
\theta=\operatorname{atan} 2\left[\left({ }^{v} \underline{v}_{s}^{w}\right)_{x},\left(\underline{v}_{s}^{v}\right)_{y}\right]
$$


Once the steer angle is known, the wheel velocity along the forward (x) axis of the wheel frame can be determined from the $\mathrm{x}$ component the wheel equation in wheel coordinates. Then the drive velocity (around the axle) can be computed using the wheel radius.

For the opposite problem of wheel sensing, measurements of wheel rotation rate provide the wheel velocities along the $\mathrm{x}$ axis of the wheel frame. Then a measurement of steer angle provides the rotation matrix needed to convert to a vector expressed in the vehicle frame.

\subsection{Forward Velocity Kinematics - Wheels to Body}

Let the term forward kinematics refer to the problem, relevant to estimation, of computing the body velocity from the wheel velocities. The wheel equation can be written in matrix form by using skew symmetric matrices to represent the cross products as a matrix products (specifically, $\underline{a} \times \underline{b}=-\underline{b} \times \underline{a}=-[\underline{b}]_{\times \underline{a}}=[\underline{b}]_{\times}^{T} \underline{a}$ ):

$$
{ }^{v} \underline{v}_{c}^{w}={ }^{v} \underline{v}_{v}^{w}+\left[\underline{r}_{s}^{v}\right]_{\times}^{T}\left({ }^{v} \underline{\omega}_{v}^{w}\right)+\left[{ }^{v} \underline{r}_{c}^{s}\right]_{\times}^{T}\left({ }^{v} \underline{\omega}_{v}^{w}\right)+\left[{ }^{v} \underline{r}_{c}^{s}\right]_{\times}^{T}\left(\underline{\omega}_{c}^{v}\right)
$$

For multiple wheels, stacking all the equations and grouping the first three terms together produces a matrix equation of the form:

$$
\underline{v}_{c}=H_{v}(\underline{\theta})\left[\begin{array}{c}
{ }^{v} \underline{\underline{v}}_{v}^{w} \\
{ }^{v} \underline{\omega}_{v}^{w}
\end{array}\right]+H_{\theta}(\underline{\theta}) \underline{\omega}_{c}^{v}=H_{v}(\underline{\theta}) \underline{V}+H_{\theta}(\underline{\theta}) \underline{\dot{\theta}}
$$

where $\underline{v}_{C}$ represents wheel velocities, and $\underline{V}$ represents the linear and angular velocity of the vehicle with respect to the ground. Both $\underline{v}_{c}$ and $\underline{V}$ are in body coordinates. $\underline{\theta}$ is the steer angles and it can include other articulations if desired. The last term in (10) is the increment to wheel velocity due to the steering rates.

\subsection{Example - Four Wheel Steer}

While the equations are linear in velocity, there can easily be more of them than there are degrees of freedom, making the problem overconstrained. Control will typically try to steer the wheels to be consistent with a single instantaneous center of rotation but errors can never be completely eliminated. A straightforward way to estimate the vehicle linear and angular angular velocity is to use the pseudoinverse - after removing the effect of steering rates:

$$
\underline{V}=H_{v}(\underline{\theta})^{+}\left(\underline{v}_{c}-H_{\theta}(\underline{\theta}) \underline{\dot{\theta}}\right)
$$

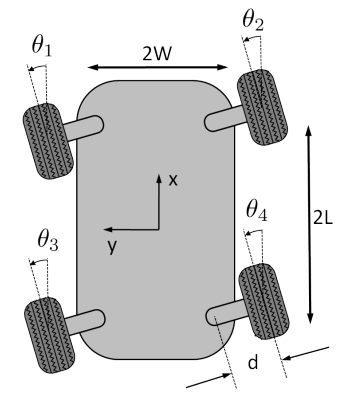

Fig. 2 A four wheel steer vehicle. Arbitrary motions are possible. 
This case (Figure 2) presents a particularly difficult example of a vehicle with four wheels which are both driven and steered (from an offset position). The equations were implemented and tested on such a vehicle. Let the velocities of the

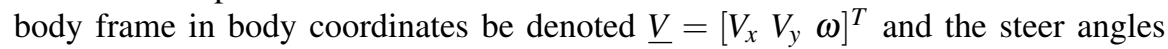
$\underline{\theta}=\left[\begin{array}{llll}\theta_{1} & \theta_{2} & \theta_{3} & \theta_{4}\end{array}\right]^{T}$. Unlike a car, this vehicle is not constrained to move in the direction it is pointed. Indeed, it can drive with any linear and angular velocity that is consistent with the wheel speed and steering limits. The steer frame centers are positioned relative to the body frame as follows:

$$
\underline{r}_{s 1}^{v}=[L W]^{T}, \underline{r}_{s 2}^{v}=[L-W]^{T}, \underline{r}_{s 3}^{v}=[-L W]^{T}, \underline{r}_{s 4}^{v}=[-L-W]^{T}
$$

The contact point offsets in the body frame depend on the steer angles. They are:

$$
{ }^{v} \underline{r}_{c 1}^{s 1}=d\left[\begin{array}{ll}
-s_{1} & c_{1}
\end{array}\right]^{T},{ }^{v} \underline{r}_{c 2}^{s 2}=d\left[s_{2}-c_{2}\right]^{T},{ }^{v} \underline{\underline{v}}_{c 3}^{s 3}=d\left[-s_{3} c_{3}\right]^{T},{ }^{v} \underline{r}_{c 4}^{s 4}=d\left[s_{4}-c_{4}\right]^{T}
$$

where $\left(s_{1}\right.$ in the vector denotes $\sin \left(\theta_{1}\right)$ etc.). If we denote the elements of these position vectors as $\underline{r}_{s}^{v}=\left[\begin{array}{ll}x y & y\end{array}\right]^{T}$ and ${ }^{v} \underline{r}_{c}^{s}=[a b]^{T}$, the set of wheel equations is as follows:

$$
\begin{aligned}
& {\left[\begin{array}{l}
v_{1 x} \\
v_{1 y}
\end{array}\right]=\left[\begin{array}{ccc}
1 & 0 & -\left(y_{1}+b_{1}\right) \\
0 & 1 & \left(x_{1}+a_{1}\right)
\end{array}\right] \underline{V}+\left[\begin{array}{cccc}
-b_{1} & 0 & 0 & 0 \\
a_{1} & 0 & 0 & 0
\end{array}\right] \underline{\dot{\theta}}} \\
& {\left[\begin{array}{l}
v_{2 x} \\
v_{2 y}
\end{array}\right]=\left[\begin{array}{ccc}
1 & 0 & -\left(y_{2}+b_{2}\right) \\
0 & 1 & \left(x_{2}+a_{2}\right)
\end{array}\right] \underline{V}+\left[\begin{array}{cccc}
0 & -b_{2} & 0 & 0 \\
0 & a_{2} & 0 & 0
\end{array}\right] \underline{\dot{\theta}}} \\
& {\left[\begin{array}{l}
v_{3 x} \\
v_{3 y}
\end{array}\right]=\left[\begin{array}{ccc}
1 & 0 & -\left(y_{3}+b_{3}\right) \\
0 & 1 & \left(x_{3}+a_{3}\right)
\end{array}\right] \underline{V}+\left[\begin{array}{cccc}
0 & 0 & -b_{3} & 0 \\
0 & 0 & a_{3} & 0
\end{array}\right] \underline{\dot{\theta}}} \\
& {\left[\begin{array}{l}
v_{4 x} \\
v_{4 y}
\end{array}\right]=\left[\begin{array}{ccc}
1 & 0 & -\left(y_{4}+b_{4}\right) \\
0 & 1 & \left(x_{4}+a_{4}\right)
\end{array}\right] \underline{V}+\left[\begin{array}{cccc}
0 & 0 & 0 & -b_{4} \\
0 & 0 & 0 & a_{4}
\end{array}\right] \underline{\dot{\theta}}}
\end{aligned}
$$

\section{Wheel Constraints}

So far, we have proposed control and estimation mechanisms that satisfy wheel slip constraints in both the forward and inverse kinematics describing the motion of the vehicle in the instantaneous terrain tangent plane. Steering and propulsion are actively controlled in a vehicle, so some measures can be taken to try to satisfy wheel slip constraints. Doing so enhances controllability and avoids the energy loss that would be associated with doing (sliding) work on the terrain.

On non-flat terrain, another constraint of interest is terrain following. Assuming an adequate suspension, wheels should neither penetrate nor rise above the terrain. Such constraints determine altitude (z), and attitude (pitch and roll). These constraints are satisfied passively by the suspensions of most vehicles, so the inverse kinematic problem of active suspension occurs less often. We will now present 
methods to incorporate both types of constraints in the context of motion prediction: the problem of estimating or predicting position and attitude by integrating the system differential equation.

\subsection{Constrained Dynamics}

We will find it convenient to formulate the WMR motion prediction problem as the integration of a differential-algebraic equation (DAE) where the constraints remain explicit. We will use a nonstandard formulation of the form:

$$
\begin{array}{r}
\dot{x}=\underline{f}(\underline{x}, \underline{u}) \\
\underline{c}(\underline{x})=\underline{0} \\
\underline{d}(\underline{x})^{T} \underline{\dot{x}}=\underline{0}
\end{array}
$$

The $m$ constraint equations in $\underline{c}$ and $\underline{d}$ are understood to be active at all times. Each element of $\underline{d}$ is a particular form of nonholonomic constraint known as a Pfaffian velocity constraint. Each specifies a disallowed direction restricting the admissible values of the state derivative. The equations in $\underline{c}$ are holonomic constraints that restrict the admissible values of the state $\underline{x}$ and therefore, through the differential equation, they ultimately restrict the state derivative as well.

Both forms of constraints are ultimately treated identically because, as is commonly performed in DAE theory, the gradient of $\underline{c}$ produces the associated disallowed directions of the holonomic constraints. It will turn out that terrain following will be expressible as holonomic constraints and wheel slip will be nonholonomic.

\subsection{Wheel Slip Constraints}

In the case of rolling without lateral slipping, the disallowed direction for the wheel is clearly aligned with the y axis of the contact point $c$ frame. However, to use the constraint in a DAE, it must be converted to an equivalent disallowed direction in state derivative space. The simplest way to do so is to write (10) in wheel coordinates thus (assuming $R_{v}^{s}=R_{v}^{c}$ ):

$$
{ }^{c} \underline{v}_{c}^{w}=R_{v}^{s} H_{v}(\underline{\theta}) \underline{V}+R_{v}^{s} H_{\theta}(\underline{\theta}) \underline{\dot{\theta}}
$$

Note that $\underline{V}$ is exactly the relevant components of the state derivative, so the first row of $R_{v}^{s} H_{v}(\underline{\theta})$ is both the gradient of the lateral wheel velocity with respect to the state derivative, and the associated disallowed direction. Note that in full-3D, a transformation from Euler angle rates to angular velocity may be required (see Section 4.1). As long as the steer angles $\underline{\theta}$ are not in the state vector, the second 
term is irrelevant, but if they are, the first row of the gradient can be extracted for these as well. If there were any other articulations in the kinematic chain from the body frame to the wheel contact point frame, they can be treated similarly.

\subsection{Terrain Following Constraints}

It is tempting to extract the $\mathrm{z}$ component of the wheel velocity in an analogous manner to produce a terrain following constraint, but the problem is slightly more complicated. It is a basic assumption that the location of the wheel contact point is known. This point is on the bottom of the wheel on flat terrain and it must be computed for uneven terrain. In any case, the axes of the $c$ frame are aligned with the wheel by assumption.

A terrain following constraint can be generated by noting that the terrain normal at the contact point is the other disallowed direction for wheel motion. Indeed, to be precise, the wheel y axis should ideally be projected onto the terrain tangent plane for lateral slip constraints as well. We can enforce terrain following by requiring the the dot product of the terrain normal and contact point velocity vectors equals zero: $\hat{n} \cdot \vec{v}_{c}^{w}=0$. Accordingly, the gradient of out-of-terrain wheel motion with respect to the state derivative $\underline{V}$ is:

$$
\underline{d}(\underline{x})^{T}={ }^{v} \hat{n}^{T} H_{v}(\underline{\theta})
$$

where ${ }^{v} \hat{n}$ is the terrain normal expressed in vehicle coordinates.

The more common approach (proposed by [16]) is to differentiate the holonomic constraints $\underline{c}(\underline{x})$ with respect to the state to obtain the gradient $\underline{c}_{x}$. The holonomic constraints are then enforced to first order by requiring that $\underline{c}_{x} \underline{\dot{x}}=\underline{0}$. Here we computed the disallowed gradient $\underline{c}_{\underline{x}}$ using vector algebra and avoided the differentiation.

\section{Results}

We present results on the Zoë rover, which previously surveyed the distribution of microscopic life in Chile's Atacama desert [15]. Zoë has four independently driven wheels on two passively articulated axles. The axles are free to rotate in both the steer $(\theta)$ and roll $(\phi)$ angles, as seen in Fig. 3. A roll averaging mechanism constrains the front and rear axle roll angles to be symmetric $\left(\phi_{f}=-\phi_{r}\right)$.

One of the authors previously developed a transform-based 3D kinematic model for the Zoë rover and applied it to control [12]. Here we re-derive the kinematic and constraint equations using the new, vector algebra formulation and apply them to estimation and simulation. 

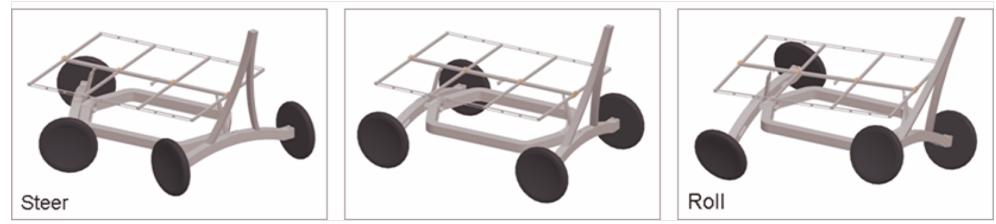

Fig. 3 Zoë's axles are free to rotate in both the steer and roll angles
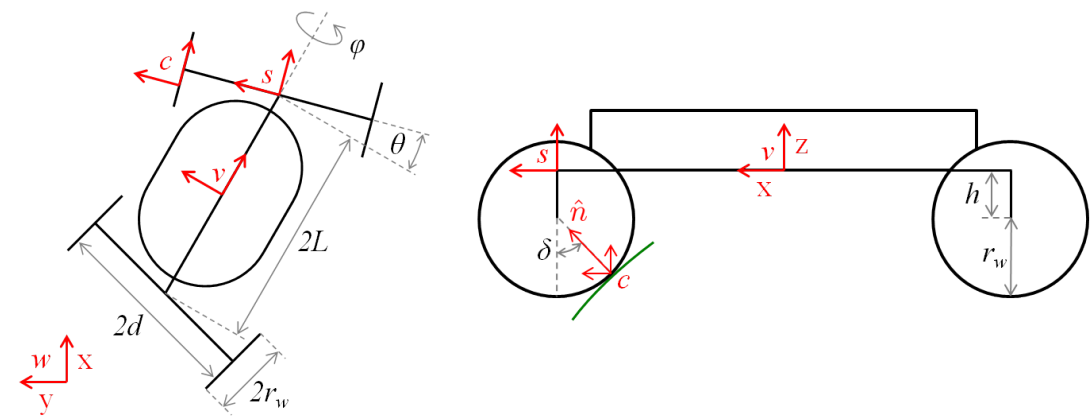

Fig. 4 A diagram of Zoë's coordinate systems, dimensions, and steering/suspension angles

\subsection{Zö̈ Forward Velocity Kinematics}

Here we present Zoë's forward velocity kinematics. We begin by defining variables in the wheel equation, arranged according to (10):

$$
{ }^{v} \underline{v}_{c}^{w}=\left[I\left[\underline{r}_{c}^{v}\right]_{\times}^{T}\right]\left[\begin{array}{c}
{ }^{v} \underline{v}_{v}^{w} \\
{ }^{v} \underline{\omega}_{v}^{w}
\end{array}\right]+\left[{ }^{v} \underline{r}_{c}^{s}\right]_{\times}^{T} \underline{\omega}_{c}^{v}
$$

The vector $\underline{r}_{c}^{v}$ is the position of the wheel contact point with respect to the vehicle, expressed in vehicle coordinates:

$$
\begin{aligned}
\underline{r}_{c}^{v} & =\underline{r}_{s}^{v}+{ }^{v} \underline{r}_{c}^{s} \\
& =\underline{r}_{s}^{v}+R_{s}^{v} \underline{\underline{s}}_{c}^{s} \\
& =\left[\begin{array}{c} 
\pm L \\
0 \\
0
\end{array}\right]+\operatorname{Rot}_{x}(\phi) \operatorname{Rot}_{z}(\theta)\left[\begin{array}{c}
-r_{w} \sin (\delta) \\
\pm d \\
-h-r_{w} \cos (\delta)
\end{array}\right]
\end{aligned}
$$

Refer to Figure 4 for the meaning of dimensions $L, r_{w}, d$, and $h$. The dimension $L$ is positive for front axle wheels and negative for rear wheels. $d$ is positive for left wheels $(1,3)$ and negative for right wheels $(2,4)$. The contact angle, $\delta$, specifies where along circumference of the wheel contact with the terrain is made.

To simulate the constrained dynamics according to (15) we must define the state vector:

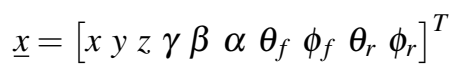


The first three states are the position of the vehicle in world coordinates $\left(\underline{r}_{v}^{w}\right)$. The second three are Euler angles (roll, pitch, and yaw), which specify the orientation of the vehicle with respect to the world frame. Let $\underline{\Omega}$ denote the vector of Euler angles: $\underline{\Omega}=[\gamma \beta \alpha]^{T}$. The last four states are the steer $(\theta)$ and roll $(\phi)$ angles for the front and rear axle joints.

We can compute $\dot{x}$ from (18), but we must first transform the angular velocities to Euler and axle angle rates as follows:

$$
\begin{gathered}
{ }^{v} \underline{\omega}_{v}^{w}=T_{\omega_{v}}\left[\begin{array}{c}
\dot{\gamma} \\
\dot{\beta} \\
\dot{\alpha}
\end{array}\right], \quad T_{\omega_{v}}=\left[\begin{array}{ccc}
1 & 0 & -s \beta \\
0 & c \gamma & s \gamma c \beta \\
0 & -s \gamma & c \gamma c \beta
\end{array}\right] \\
\left.{ }^{s} \underline{\omega}_{s}^{v}=T_{\omega_{s}}\left[\begin{array}{c}
\dot{\theta} \\
\dot{\phi}
\end{array}\right], \quad T_{\omega_{s}}=\left[\begin{array}{l}
0 \\
0 \\
1
\end{array}\right]\left(R_{v}^{s}\left[\begin{array}{l}
1 \\
0 \\
0
\end{array}\right]\right)\right]
\end{gathered}
$$

The matrix $T_{\omega_{v}}$ (for the Euler angle convention where $R_{v}^{w}=\operatorname{Rot}_{z}(\alpha) \operatorname{Rot}_{y}(\beta) \operatorname{Rot}_{x}(\gamma)$ ) is widely used in navigation [14]. Given the transforms in (23) and (24) and combining wheel equations for all four wheels, we obtain:

$$
\begin{aligned}
& \underline{v}_{c}=H_{v}(\underline{\theta}) \underline{V}^{\prime}+H_{\theta}(\underline{\theta}) \underline{\dot{\theta}}
\end{aligned}
$$

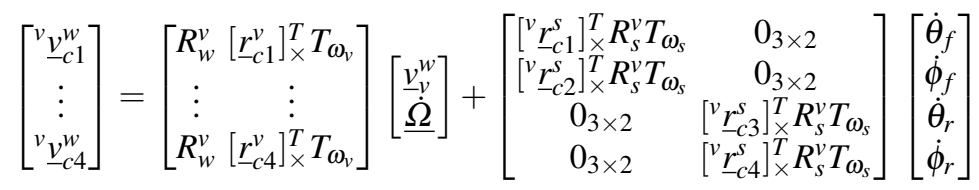

Each wheel corresponds to three rows of (26). Note that $\underline{V}^{\prime}$ differs from $\underline{V}$ as defined in (10) because it contains linear velocities in world coordinates and Euler angle rates $\underline{\dot{\Omega}}$. Note also that variables containing $s$ in the superscript or subscript are different for the front and rear axles, i.e. for wheels $(1,2)$ and $(3,4)$.

Because Zoë's steering and suspension joints are passive, it is necessary in simulation (or prediction) contexts to solve for the joint angle rates $\underline{\dot{\theta}}$ simultaneously with the vehicle velocity $\underline{V}^{\prime}$ :

$$
\underline{v}_{c}=\left[H_{v}(\underline{\theta}) H_{\theta}(\underline{\theta})\right]\left[\begin{array}{l}
\underline{V}^{\prime} \\
\dot{\dot{\theta}}
\end{array}\right]=H(\underline{\theta}) \dot{\dot{x}} .
$$

The system is overdetermined and can be solved for $\underline{\dot{x}}$ using the pseudoinverse.

\subsection{Zö̈ Constraint Equations}

As formulated, there are nine total constraints on Zoë's forward kinematics. As explained in Section 3.2 the nonholonomic, no-lateral-slip constraints are enforced by disallowing wheel velocity along the y axis of the $c$ frame for each wheel. To com- 
pute the constraint for a single wheel, we extract the corresponding 3 rows of (27) and left multiply by $R_{v}^{s}\left(=R_{v}^{c}\right)$ to convert to wheel coordinates:

$$
{ }^{c} \underline{v}_{c}^{w}=R_{v}^{s} H_{c}(\underline{\theta}) \dot{\dot{x}}
$$

where $H_{c}$ denotes the three rows of $H$ corresponding to the chosen wheel. The disallowed direction in state space $\underline{d}(\underline{x})^{T}$ is simply the second row of $R_{v}^{s} H_{c}(\underline{\theta})$. Because, in this case, left and right wheels on the same axle generate identical no-lateral-slip constraints, one redundant constraint may be eliminated per axle.

As explained in Section 3.3, the four holonomic terrain following constraints are enforced, to first order, by disallowing wheel velocity in the terrain-normal direction for each wheel. Given that the dot product ${ }^{v} \hat{n} \cdot{ }^{v} \underline{v}_{c}^{w}$ must be zero, where ${ }^{v} \hat{n}$ is the terrain normal vector expressed in vehicle (or body) coordinates, the disallowed direction in state space is ${ }^{v} \hat{n}^{T} H_{c}$.

The roll-averaging mechanism generates one additional holonomic constraint that $\phi_{f}+\phi_{r}=0$. This is enforced to first-order by constraining $\dot{\phi}_{f}+\dot{\phi}_{r}=0$.

\subsection{Terrain Following Experiment}

Here we present the results of a terrain-following experiment. Zoë is commanded to drive straight at $0.15 \mathrm{~m} / \mathrm{s}$ while its left wheels traverse a ramp obstacle $(1.71 \mathrm{~m}$ length $\times 0.41 \mathrm{~m}$ height, $36^{\circ}$ slope) that causes the body to roll. A simple proportional controller drives the front and rear steer angles to zero degrees.

Results are shown (from left to right in Figure 6) for a physical experiment, a Lagrangian dynamics simulation (implemented using Open Dynamics Engine), and a kinematic simulation using the vector algebra formulation presented here. Note that all three plots match closely; the same changes in suspension and steer angles are observed as the front and rear wheels encounter the obstacle, and both simulations correctly predict a terminal heading error of approximately $2.5^{\circ}$. The kinematic simulation, however, is computationally much cheaper than the full dynamics simulation. Surface contact parameters were tuned to minimize wheel slip in the dynamic simulation. Minor disagreements between the physical experiment and simulations are due to unmodeled peculiarities in Zoë's construction, such as hysteresis in the roll-averaging mechanism.

\section{Conclusion}

This paper has shown how the Transport Theorem provides the basis for modeling the kinematic relationships between the body velocities and the wheel velocities of a wheeled mobile robot. Our formulation produces the solution for the general three dimensional case for arbitrary robot articulations and arbitrary terrain. In contrast to all prior work in WMR kinematics, we use an intrinsic velocity transformation in coordinate system independent form. This approach has the key advantage of 

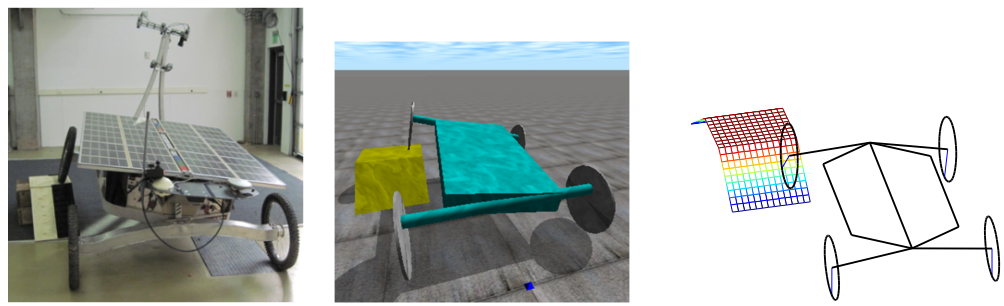

Fig. 5 Photographs and screenshots of the Zoë rover captured during (from left to right) a physical experiment, Lagrangian dynamics simulation, and kinematic simulation.
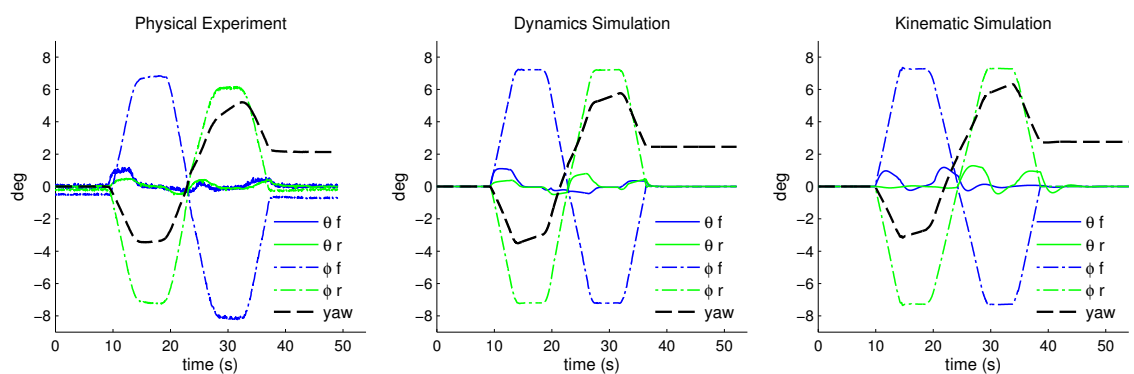

Fig. 6 Plots of steering $(\theta)$, suspension $(\phi)$, and vehicle yaw angles vs. time recorded during (from left to right) a physical experiment, Lagrangian dynamics simulation, and kinematic simulation using our presented vector algebra model.

eliminating the need to differentiate the relevant pose transforms in order to produce a mechanism Jacobian for each wheel. Furthermore, the resultant equation for wheel motion also provides a very convenient basis for imposing constraints for wheel slip and terrain following in a differential-algebraic system. Although we have not justified it here, this turns out to enable efficient predictor-corrector integration.

We have also shown advantages relative to a full second order dynamics model. Our formulation needs to be integrated only once but it satisfies the same constraints and those constraints ultimately determine the trajectory followed. Furthermore, because we express our constraints explicitly, our formulation permits them to be arbitrary. We have shown how to model an example of passive articulations here, but we can also accomodate models of how wheels are actually slipping both longitudinally and laterally. In short, ease of derivation, faster-than-real-time computation, and highly realistic motion make our formulation a natural choice for the modeling of any wheeled mobile robot in arbitrary terrain. 


\section{Acknowledgments}

This research was made with U.S. Government support under and awarded by the DoD, Air Force Office of Scientific Research, National Defense Science and Engineering Graduate (NDSEG) Fellowship, 32 CFR 168a. The NASA funded Life in the Atacama project (NAG5-12890) supported development of the Zoë rover.

\section{References}

1. Alexander, J.C., Maddocks, J.H.: On the kinematics of wheeled mobile robots. International Journal of Robotics Research 8(5), 15-27 (1989)

2. Campion, G., Bastin, G., D'Andrea-Novel, B.: Structural properties and classification of kinematic and dynamic models of wheeled mobile robots. IEEE Transactions on Robotics 12(1), 47-62 (1996)

3. Chakraborty, N., Ghosal, A.: Kinematics of wheeled mobile robots on uneven terrain. Mechanism and Machine Theory 39(12), 1273-1287 (2004)

4. Choi, B.J., Sreenivasan, S.V.: Gross motion characteristics of articulated mobile robots with pure rolling capability on smooth uneven surfaces. IEEE Transactions on Robotics 15(2), 340-343 (1999)

5. Cox, I.J., Wilfong, G.T.: Autonomous Robot Vehicles. Springer-Verlag, New York (1990)

6. Howard, T., Kelly, A.: Optimal rough terrain trajectory generation for wheeled mobile robots. International Journal of Robotics Research 26(2), 141-166 (2007)

7. Kim, D.S., Lee, H.C., Kwon, W.H.: Geometric kinematics modeling of omni-directional autonomous mobile robot and its applications. IEEE International Conference on Robotics and Automation pp. 2033-2038 (2000)

8. Lamon, P., Siegwart, R.: 3D position tracking in challenging terrain. The International Journal of Robotics Research 26(2), 167-186 (2007)

9. Luh, J.Y.S., Walker, M.W., Paul, R.P.C.: On-line computational scheme for mechanical manipulators. J. Dyn. Sys., Meas., Control 102(2), 69-76 (1980)

10. Muir, P.F., Neuman, C.P.: Kinematic modeling of wheeled mobile robots. CMU Robotics Institute Tech. Report (1986)

11. Rajagopalan, R.: A generic kinematic formulation for wheeled mobile robots. Journal of Robotic Systems 14(2), 77-91 (1997)

12. Seegmiller, N., Wettergreen, D.: Control of a passively steered rover using 3-D kinematics. In: IEEE/RSJ International Conference on Intelligent Robots and Systems (2011)

13. Tarokh, M., McDermott, G.: Kinematics modeling and analyses of articulated rovers. IEEE Transactions on Robotics 21(4), 539-553 (2005)

14. Titterton, D., Weston, J.: Strapdown Inertial Navigation Technology, 2nd. edn. AAAI (2004)

15. Wettergreen, D., et al.: Second experiments in the robotic investigation of life in the Atacama desert of Chile. In: Proc. 8th International Symposium on Artificial Intelligence, Robotics and Automation in Space (2005)

16. Yun, X., Sarkar, N.: Unified formulation of robotic systems with holonomic and nonholonomic constraints. IEEE Transactions on Robotics 14(4), 640-650 (1998) 